\title{
Hot horizontal branch stars: Predictions for mass loss
}

\section{Winds, rotation, and the low gravity problem}

\author{
Jorick S. Vink ${ }^{1,2}$ and Santi Cassisi ${ }^{3}$ \\ 1 Imperial College, Blackett Laboratory, Prince Consort Road, London SW7 2BZ, UK \\ 2 Astronomical Institute, Utrecht University, PO Box 80000, 3508 TA Utrecht, The Netherlands \\ 3 I.N.A.F. - Osservatorio Astronomico di Collurania, Via M. Maggini, 64100 Teramo, Italy
}

\author{
Received 21 May 2002 / Accepted 17 June 2002
}

\begin{abstract}
We predict mass-loss rates for the late evolutionary phases of low-mass stars, with special emphasis on the consequences for the morphology of the Horizontal Branch (HB). We show that the computed rates, as predicted by the most plausible mechanism of radiation pressure on spectral lines, are too low to produce $\mathrm{EHB} / \mathrm{sdB}$ stars. This invalidates the scenario recently outlined by Yong et al. (2000) to create these objects by mass loss on the HB. We argue, however, that mass loss plays a role in the distribution of rotational velocities of hot HB stars, and may - together with the enhancement of heavy element abundances due to radiative levitation - provide an explanation for the so-called "low gravity" problem. The mass loss recipe derived for hot $\mathrm{HB}$ (and extreme $\mathrm{HB}, \mathrm{sdB}$, sdOB) stars may also be applied to post-HB (AGB-manqué, UV-bright) stars over a range in effective temperatures between $12500-40000 \mathrm{~K}$.
\end{abstract}

Key words. stars: horizontal-branch - subdwarfs - stars: mass-loss - stars: winds, outflows - stars: evolution - galaxy: globular clusters: general

\section{Introduction}

Over the last decades, both observational and theoretical efforts have been devoted to the investigation of the observed distribution of stars along the Horizontal Branch (HB) of galactic Globular Clusters (GCs). Although canonical stellar evolution theory has provided a general consensus on the evolutionary phase corresponding to the HB sequence, and convincingly demonstrated that its morphology is most strongly affected by cluster metallicity (the "first" parameter; Sandage \& Wallerstein 1960), many problems remain. The most striking controversy involves the wide variety of HB morphologies among clusters with similar metallicities (the "second parameter" problem; Sandage \& Wildey 1967; van den Bergh 1967). Candidate second parameters are cluster age (e.g. Lee et al. 1994 and references therein), mass loss along the Red Giant Branch (RGB) (Catelan et al. 2001 and references therein), rotation and deep helium mixing (Sweigart 1997), dynamical interactions involving binaries and even planets (Soker 1998), as well as environmental effects in highdensity environments (Fusi Pecci et al. 1993).

The identification of the second parameter is especially relevant to the formation of Extremely blue HB (EHB) stars, which are thought to be responsible for the ultraviolet upturn

Send offprint requests to: J. S. Vink, e-mail: jvink@ic. ac .uk phenomenon in elliptical galaxies (Greggio \& Renzini 1990; Dorman et al. 1995). The presence of EHB stars as blue "tails" in clusters (Ferraro et al. 1998; Piotto et al. 1999), as well as $\mathrm{sdB} / \mathrm{sdO}$ stars in the field (Greenstein 1971; Green et al. 1986), has inspired modern-day research to explain their formation both through mechanisms that produce high mass loss along the RGB (Soker et al. 2001 and references therein), as well as through binarity (Mengel et al. 1976; Heber et al. 2002).

Further puzzles in HB morphology concern the issues of HB "gaps" (Newell 1973) - specific regions along the branch that are significantly underpopulated ${ }^{1}$, and a relatively new, unexplained, but ubiquitous feature is the so-called Strömgren $u$-jump at an effective temperature of $T_{\text {eff }} \simeq 11000 \mathrm{~K}$ (Grundahl et al. 1999), possibly coinciding with a jump in $\log g$ (Moehler et al. 1995), and an unexplained absence of fast rotators above this temperature (Behr et al. 2000; Recio-Blanco et al. 2002).

As discussed by Grundahl et al. (1999), the Strömgren $u$-jump may be due to atmospheric diffusion by radiative

\footnotetext{
${ }^{1}$ It has been claimed that the positions of the gaps along the HB in different galactic GCs are the same within current empirical uncertainties (Ferraro et al. 1998), however it is not clear whether these gaps mark regions with specific effective temperatures, or whether they correspond to constant mass loci (Piotto et al. 1999). Note that Catelan et al. (1998) have challenged the existence of gaps at the same positions in all clusters.
} 
levitation of heavy elements, as both Glaspey et al. (1989) and Behr et al. (1999) found striking abundance anomalies in blue HB stars, with iron enhancements of up to three times the solar value. Moehler et al. (2000) have shown that the enhancement of heavy elements in spectroscopic analyses may partially solve the problem of the anomalously low gravities along the blue $\mathrm{HB}$, but the discrepancy is still present at the level of $\Delta \log g \approx 0.1 \mathrm{dex}$ for stars in the range $15000<T_{\text {eff }}<$ $20000 \mathrm{~K}$. Even more so, the first two mentioned HB features the blue tails and the gaps - are still an enigma ${ }^{2}$, and it is not at all obvious whether they originate from a mechanism working in a prior evolutionary phase (on the RGB) or if they are due to a process working "in situ" once the star has settled on the HB. One of the options that may help in explaining the abovementioned problems is mass loss on the HB.

It is worth mentioning that the colour width of the hottest gap is so small that changes of the order of a few times $10^{-3} M_{\odot}$ in the total mass are capable to move the star away from its initial location, far enough as to produce an underpopulated region in the H-R diagram (HRD). For this to occur one needs to identify a mass-loss mechanism which efficiency rapidly increases at the specific effective temperatures of the gap.

The hypothesis that a mass-loss mechanism may be at work during the HB evolution was first entertained by Wilson \& Bowen (1984). They suggested that an increased mass-loss efficiency, when crossing the RR Lyrae instability strip, could provide an explanation for the $\mathrm{HB}$ mass distribution in a more natural way than the alternative of a stochastic variation in the amount of mass lost during the prior RGB phase. The topic of mass loss on the HB was further addressed by Koopmann et al. (1994), but they concluded that constant mass loss in the RR Lyrae strip was incapable of providing an explanation for the HB mass dispersion or the RR Lyrae period change distribution. Additionally, mass loss during the central He-burning phase was suggested by Michaud et al. (1985) and Bergeron et al. (1988) in order to explain the large silicon underabundances in some HB stars. More recently, Yong et al. (2000) performed accurate evolutionary computations with mass loss, and suggested that mass-loss rates of the order of $10^{-9}-10^{-10} M_{\odot} \mathrm{yr}^{-1}$ for HB stars in the metal-rich cluster NGC 6791 can force these stars to move to a bluer position and thus lead to the production of EHB stars. If correct, this scenario could provide an explanation for the presence of extended blue tails along the HB of some metal-rich GCs, such as NGC 6441 and NGC 6388 (Rich et al. 1997), although it would not be able to explain the upward sloping of the HB in these clusters (Raimondo et al. 2002 and references therein). The main problem with the proposed scenario, however, is that no physical mechanism for mass loss was proposed and that the adopted mass-loss rates were completely "ad hoc", as there are neither observational data indicative of mass loss on the $\mathrm{HB}$ available, nor any predictions.

Our aim in the present paper is to alleviate current shortcomings by computing radiation-driven wind models and

\footnotetext{
${ }^{2}$ Note that Brown et al. (2001) and Sweigart et al. (2002) have provided a theoretical framework which could explain the hot gap in the HB of NGC 2808.
}

mass-loss rates for low-mass blue stars, and to subsequently investigate their influence on HB evolutionary models. Blue HB stars are located in a region of the HRD, where the stars are hot (with $T_{\text {eff }}$ between 10000 and $35000 \mathrm{~K}$ ), and relatively bright, and radiation pressure forces can therefore be considered a natural driving mechanism. Although there may be other processes that could possibly drive a wind, such as pulsations ${ }^{3}$, all other wind-driving options are much less well-understood than radiation pressure on spectral lines. Radiation-driven wind models have been developed in the 1970s by Lucy \& Solomon (1970) and Castor et al. (1975). In more recent days, the models have been very successful in predicting the values observed in O supergiants (Vink et al. 2000). The direct application of these predictions to HB stars, such as the use of the mass-loss recipe provided by Vink et al. (2000) would however involve a rather large and dangerous extrapolation by four orders of magnitude in stellar luminosity.

As far as the "gaps" along the HB are concerned, radiationdriven wind models for OB supergiants predict that the efficiency of mass loss jumps strongly by a factor of five at spectral type B1 (Vink et al. 1999, 2000). This is close to the position where the evidence for a gap in HB morphology is strongest. A mass-loss rate of the order of $10^{-10}-10^{-11} M_{\odot} \mathrm{yr}^{-1}$ could be sufficient to explain the presence of the gap located at $T_{\text {eff }} \simeq 20000 \mathrm{~K}$; given an HB evolutionary timescale of $\approx 10^{8}$ years with mass loss at this rate leads to a total amount of a few times $10^{-3} M_{\odot}$ sufficient to move an HB star by $\approx 1000 \mathrm{~K}$, and so creating a "gap".

The above-mentioned issues, i.e. the presence of EHB stars, gaps, and anomalous abundances in HBs and sdB stars, prompted us to compute radiation-driven wind models for HB stars; to predict mass-loss rates for these objects, and subsequently explore their influence on evolutionary models. The mass loss computations may also provide valuable ingredients for HB angular momentum evolution and chemical separation calculations of sdB stars (see Unglaub \& Bues 2001).

The outline of the paper is as follows. In the next section we describe the approach used for computing mass-loss rates, as well as the assumptions adopted in the numerical computations; in Sect. 3 we discuss the results concerning the mass-loss efficiency, where the dependence of $\dot{M}$ on the main evolutionary parameters, the luminosity, effective temperature and stellar mass, as well as stellar metallicity, is presented. In Sect. 4, we provide an analytical relation for $\dot{M}$ as a function of the quoted parameters, which is useful for computing the mass-loss rates in evolutionary computations, and we investigate the effects of our recipe on HB stellar evolution (Sect. 5). In Sect. 6, we study the implications of mass loss regarding the "zoo" of problems in $\mathrm{HB}$ morphology that occur for effective temperatures larger than $\simeq 10000 \mathrm{~K}$, in particular the effects of mass loss on

\footnotetext{
3 Recently a new class of variable stars has been discovered in the field, the so-called EC14026 (Kilkenny et al. 1997), which have been identified as hot HB stars and their progeny. Even though no clear identification of similar variables in GCs have been obtained, there is no a priori reason for the lack of this kind of pulsation among cluster HB stars.
} 
rotational velocities and the $\log g$ jump. Final remarks and conclusions will close the paper.

\section{Method and assumptions in computing $\dot{M}$}

We compute mass-loss rates for HB stars under the hypothesis that radiation pressure on spectral lines drives a stellar wind on the HB. Although this does not imply that there are no other physical mechanisms operating during the HB phase that could possibly drive a wind, it is the most sophisticated wind theory known, and it has been very successful in explaining the observed mass-loss rates of hot massive stars.

\subsection{The Monte Carlo method to calculate $\dot{M}$}

The description of the radiative wind driving with our method is based on a Monte Carlo technique that was first introduced by Abbott \& Lucy (1985). This approach naturally accounts for photon-interactions with different metal ions, as the photons try to escape from the stellar wind. In the Monte Carlo model used here (MC-WIND, de Koter et al. 1997; Vink et al. 1999), the momentum deposition is calculated using the Sobolev approximation by following the fate of a large number of photons that are released from below the stellar photosphere. To obtain a consistent solution, several wind models are calculated to find the mass-loss rate that is consistent with the radiative acceleration (see also Lucy \& Abbott 1993).

The calculation of radiation pressure with this method requires the input of a model atmosphere. The model atmospheres used in this study are the non-LTE unified Improved Sobolev Approximation code (ISA-WIND), which treats the photosphere and wind in a unified manner (distinct from the socalled "core-halo" approaches). For details of the code we refer the reader to de Koter et al. $(1993,1997)$. The chemical species that are explicitly calculated in non-LTE are $\mathrm{H}, \mathrm{He}, \mathrm{C}, \mathrm{N}, \mathrm{O}$, and $\mathrm{Si}$. The iron-group elements are treated in a generalised version of the "modified nebular approximation" (Lucy 1987, 1999).

\subsection{Assumptions in the models}

The model depends upon the assumption that the plasma behaves as a single fluid. As long as a large number of collisions between the accelerating (C,N,O, and Fe-group) and non-accelerating $(\mathrm{H}$ and $\mathrm{He})$ particles ensures a strong coupling, one can safely treat the wind as a single fluid. A simple condition for this so-called "Coulomb coupling" is given by Lamers \& Cassinelli (1999, p. 193):

$$
\frac{L_{*} v}{\dot{M}}<5.9 \times 10^{16}
$$

where $L_{*}$ is in $L_{\odot}, v$ is in $\mathrm{km} \mathrm{s}^{-1}$ and $\dot{M}$ is in $M_{\odot} \mathrm{yr}^{-1}$. Although this Coulomb condition is easily satisfied for the dense winds of massive $\mathrm{O}$ stars, it may break down for weaker winds, such as the winds from main-sequence A and B stars (Springmann \& Pauldrach 1992; Porter \& Drew 1995; Babel 1996; Krticka \& Kubát 2000). However, using typical values for an EHB star, $\log L_{*}=1.3 ; v=600 \mathrm{~km} \mathrm{~s}^{-1}$; and $\log \dot{M}=-11.70$ (see computations in Sect. 3), we find that the ratio $\left(L_{*} v / \dot{M}\right)$ from Eq. (1) is ten times smaller than the quoted value of $5.9 \times 10^{16}$, indicating that the condition of Coulomb Coupling is fulfilled. It remains yet to be seen if the use of the Sobolev approximation is valid for weaker winds (see Owocki \& Puls 1999).

\subsection{The adopted parameters}

Effective Temperatures. The models have effective temperatures between 12500 and $35000 \mathrm{~K}$ with stepsizes of $2500 \mathrm{~K}$. We have checked this choice of gridding by computing additional models at intermediate temperatures, which showed our initial resolution to be entirely adequate.

Luminosities and masses. The values for the stellar luminosity $(L)$ and mass $(M)$ were taken from evolutionary models (Cassisi \& Salaris 1997; Zoccali et al. 2000) for HB stars computed under different assumptions regarding the initial chemical composition.

Metal abundances. The adopted heavy elements distribution corresponds to the solar scaled one provided by Anders \& Grevesse (1989), adopting $Z_{\odot}=0.019$. For non-solar metallicity $Z$, the helium $(Y)$ and hydrogen $(X)$ abundances are adjusted in the following way:

$Y=Y_{\mathrm{p}}+\left(\frac{\Delta Y}{\Delta Z}\right) Z$

where the primordial helium abundance $Y_{\mathrm{p}}=0.24$ (Audouze 1987) and $(\Delta Y / \Delta Z)=3$ (Pagel et al. 1992). $X$ is then simply given by:

$X=1-Y-Z$.

Velocity law. We calculated $\dot{M}$ for wind models with a $\beta$-type velocity law for the accelerating part of the wind:

$v(r)=v_{\infty}\left(1-\frac{R_{*}}{r}\right)^{\beta}$.

Below the sonic point, a smooth transition from this velocity structure is made to the velocity that follows from the photospheric density structure. A value of $\beta=1$ was adopted in the accelerating part of the wind. This is a typical value for $\mathrm{OB}$ supergiants (Groenewegen \& Lamers 1989; Puls et al. 1996), but future mass loss observations of HB stars should be able to show whether or not this is also an adequate description for these low mass objects. Note that it has been demonstrated that over a $\beta$ range $0.7-1.5$ the $\dot{M}$ predictions are insensitive to the adopted value of $\beta$ (Vink et al. 2000). We further assume a ratio $v_{\infty} / v_{\text {esc }}=1.0$, as roughly indicated by $v_{\infty}$ observations for a handful of SdO stars by Hamann et al. (1981) and Howarth (1987).

\section{The mass-loss predictions}

Using the procedure described in Sect. 2.1, we have calculated mass-loss rates as a function of $T_{\text {eff }}$ with temperatures in the 


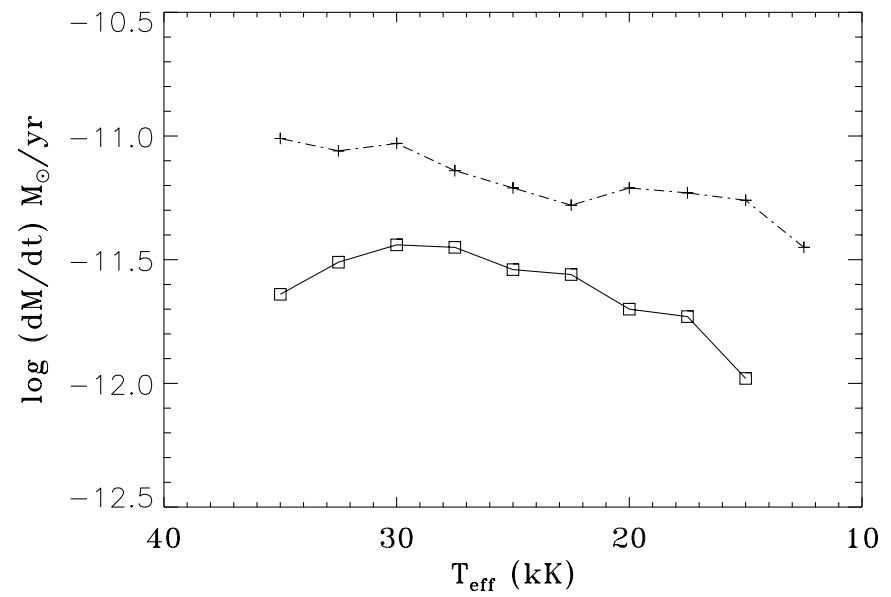

Fig. 1. Mass loss predictions for $\mathrm{HB}$ stars as a function of effective temperature. The solid line represents the computations for a solar metallicity. The dotted line is for a metallicity three times solar. The models are calculated for a constant mass of $M=0.5 M_{\odot}$ and $\log \left(L / L_{\odot}\right)=1.5$.

range between 12500 and $35000 \mathrm{~K}$. This was performed for luminosities in the range $\log \left(L_{*} / L_{\odot}\right)=1.3-1.7$ and masses in the range $M_{*}=0.5-0.7 M_{\odot}$.

\subsection{Mass loss as a function of $T_{\text {eff }}$}

The results of our predictions of $\mathrm{HB}$ mass loss as a function of effective temperature are presented in Fig. 1. The solid line represents the computations for solar metallicity. To check the generality of this behaviour we have also computed mass loss as a function of $T_{\text {eff }}$ for somewhat different input parameters, represented by the dotted line, for a metallicity three times solar. In both cases there appears to be a slight decrease of mass loss as a function of decreasing effective temperature. This can be attributed to the gradual shift of the flux maximum towards longer wavelengths, and as the number of lines present in the spectrum is smaller at higher wavelengths, the line acceleration decreases, reducing the mass-loss rate. Superimposed on this, one may have expected to see jumps, where $\dot{M}$ could increase due to recombinations of important line-driving ions. As mentioned earlier, for OB supergiants the mass loss increases steeply by a factor of five due to the recombination of $\mathrm{Fe}$ IV to Fe III at spectral type B1.

Figure 1 however, indicates that these so-called "bistability" jumps are absent for HB stars. This is probably due to the lower wind densities in $\mathrm{HB}$ stars in comparison to $\mathrm{OB}$ supergiants. In fact, these HB computations (for solar metallicity) are more comparable to the OB supergiant calculations at very low metallicities. Vink et al. (2001) have shown that at lower wind densities, the winds are no longer driven by $\mathrm{Fe}$, but that the line driving by CNO-like elements takes over, and the dramatic recombinations are much less pronounced, or even absent at the temperatures under consideration here.

We conclude, that bi-stability jumps are not present for HB stars and we apply a fit through our computed datapoints over the complete temperature range. This temperature dependence

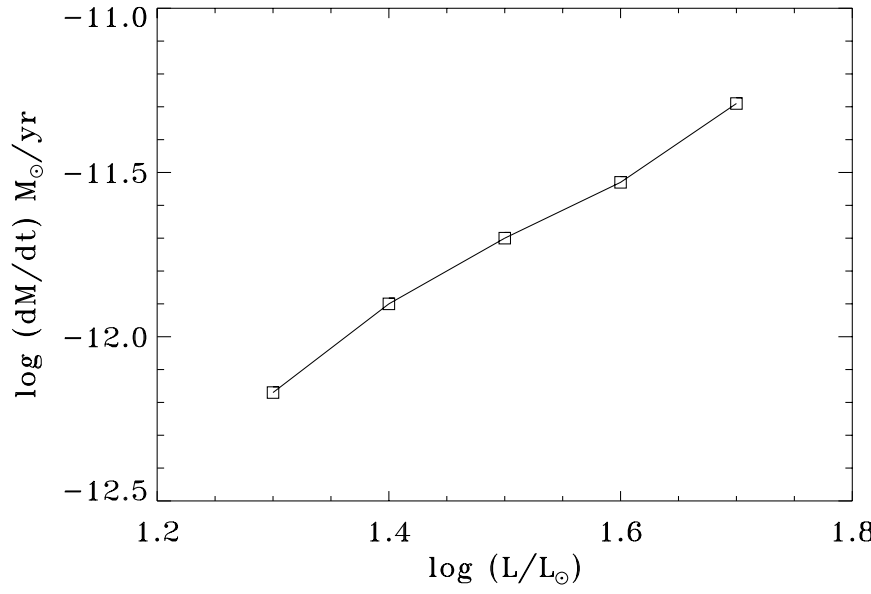

Fig. 2. Mass loss predictions for HB stars as a function of stellar luminosity. These models are calculated for a constant mass of $M=$ $0.5 M_{\odot}, T_{\text {eff }}=20000 \mathrm{~K}$, and solar metallicity.

of mass loss is later incorporated into our mass loss recipe for HB stars (Sect. 4).

\subsection{Mass loss as a function of $L, M$ and $Z$}

Predictions of HB mass loss as a function of other stellar parameters, namely $L, M$, and $Z$ have also been performed. As an example, the results for mass loss as a function of $L$ are shown in Fig. 2. The results of $\dot{M}$ as a function of $L, M$ and $Z$ do not yield any surprises. As expected, mass loss increases for increasing $L$ and $Z$, but decreasing $M$. Although the behaviour is qualitatively similar to the $\mathrm{O}$ star recipe in Vink et al. (2000), the dependencies are somewhat different. The values of these dependencies and mass-loss rates are discussed in the next section.

\section{Mass loss recipe}

In this section, we present a mass loss recipe for HB stars as a function of basic stellar parameters. To obtain the recipe we have determined four separate dependencies, and checked if they were independently applicable. As this was found to be the case, we have combined the four independent parameters from Sects. 3.1 and 3.2 and constructed the following analytical relationship for HB mass loss:

$$
\begin{aligned}
\log \dot{M}= & -11.70( \pm 0.08) \\
& +1.07( \pm 0.32) \log \left(T_{\mathrm{eff}} / 20000\right) \\
& +2.13( \pm 0.09)\left(\log L_{*}-1.5\right) \\
& -1.09( \pm 0.05) \log \left(M_{*} / 0.5\right) \\
& +0.97( \pm 0.04) \log \left(Z_{*}\right)
\end{aligned}
$$

derived for:

$$
\begin{aligned}
& 12500 \leq T_{\text {eff }} \leq 35000 \mathrm{~K} \\
& 1.3 \leq \log L_{*} \leq 1.7 \\
& 0.5 \leq M_{*} \leq 0.7 \\
& 0.1 \leq Z_{*} \leq 10
\end{aligned}
$$


where $T_{\text {eff }}$ is in Kelvin and $L_{*}, Z_{*}$, and $M_{*}$ are all given in solar units. Note that the computations have been performed for the case $v_{\infty}=v_{\text {esc }}$ (see Sect. 2.3). As discussed in Vink \& de Koter (2002), $\dot{M}$ is maximal for this value, and Eq. (5) therefore provides an upper limit to the mass-loss rate. In case $v_{\infty}$ is not equal to $v_{\text {esc }}$, one can use the $\left(v_{\infty} / v_{\text {esc }}\right)$ dependence of $\dot{M}$ as derived in Vink et al. (2000) for OB stars. The root-mean-square (rms) difference between the results following from Eq. (5) and the actual model computations is $0.08 \mathrm{dex}$ in $\log \dot{M}$. This implies that the HB formula yields a good representation of the actual HB mass loss computations.

Alternatively, if we put the HB stellar parameters into the recipe of Vink et al. (2000) for massive O stars (and keep $v_{\infty}$ fixed to $v_{\text {esc }}$ ), we find an rms difference of 0.44 dex in the mass loss, leading to the conclusions that an extrapolation of the O star recipe by four orders of magnitude in stellar luminosity would have resulted in mass-loss rates that are systematically too high by about a factor of two. One may still wonder whether the HB mass loss recipe of Eq. (5) is also applicable to stars with stellar parameters for which the recipe was not specifically derived. To check whether Eq. (5) may safely be used over a wider range in stellar parameters, we have performed mass loss calculations for the winds of post-HB stars and compared these actual calculations with the results from the recipe. We find that the mass loss recipe may be used for other classes of low-mass blue objects as well, as long as the desired accuracy is within a factor of $\sim 2$. As there are hardly any mass-loss predictions available for these types of objects either, the HB formula may be applied to all hot, low-mass stars, of the types: HB, EHB, sdB, sdOB, post-HB, AGB-manqué, UV-bright stars, and extreme helium stars, as long as their effective temperatures are not significantly higher than $\sim 40000 \mathrm{~K}$. This because existing mass loss calculations, such as the ones presented here, but also those by Pauldrach et al. (1988) for Central Stars of Planetary Nebulae have the problem that line lists become incomplete with respect to higher ionisation stages. Note that we do not expect problems with extrapolating Eq. (5) to effective temperatures as low as $\approx 8000 \mathrm{~K}$. A computer routine of the HB mass loss recipe is available upon request or on the $\mathrm{Web}^{4}$.

\section{Mass loss effects on HB evolution}

\subsection{Assumptions in evolutionary tracks and outer boundary conditions}

In order to check the effects of the computed mass-loss recipe on evolutionary tracks of HB stars, we have computed two series of models, identical in every way, except that one set incorporates the mass-loss recipe, while the occurrence of mass loss is neglected in the other. All models have been computed using the FRANEC evolutionary code (Cassisi \& Salaris 1997; Castellani et al. 1997 and references therein). As far as the adopted physical input parameters as well as the treatment of convection during the central He-burning phase are concerned, we refer the interested reader to the papers by Cassisi \& Salaris (1997) and Zoccali et al. (2000). The treatment of outer

\footnotetext{
${ }^{4}$ http://astro.ic.ac.uk/ jvink/
}

boundary conditions is performed, as usual, by adopting a $T(\tau)$ relation (Krishna-Swamy 1966).

To check the validity of this assumption for hot HB stars, we have computed a large grid of model atmospheres that provide more accurate descriptions of the thermal stratification of the atmospheres of these stars, and investigated whether a different treatment of the outer boundary conditions influences the evolutionary output parameters. To this end, we computed several HB models for different metallicities by adopting in one case the $T(\tau)$ relation from Krishna-Swamy, and in the other the boundary conditions provided by the more sophisticated model atmosphere computations. The model atmospheres used for this test were the non-LTE ISA-WIND models of de Koter et al. (1993, 1997) with negligible mass loss, as well as the hydrostatic LTE, line-blanketed models of Kurucz (1993). In the model atmosphere cases, the connection between the atmospheres and the internal structures has been fixed at $\tau_{\text {Ros }}=10$. Note that we have verified that the results obtained are not affected by this choice of matching point between stellar atmosphere and internal regions. Our numerical experiments clearly showed that the stellar properties such as the effective temperature, are not significantly affected by the assumptions made concerning the outer boundary conditions. This is because the $T(\tau)$ approach provides an estimate of the thermal stratification in the stellar atmosphere that is in good agreement with those provided by more accurate model atmospheres computations, as can be seen in Fig. 3, where we have plotted the thermal stratifications provided by the $T(\tau)$ method, and the model atmospheres quoted above, for values of the effective temperature and surface gravity suitable for hot HB stars. These results imply that canonical theoretical predictions of effective temperatures of hot HB stars can be considered to be robust.

\subsection{HB Evolution with mass loss for metal rich clusters}

In order to maximise the effects of mass loss on the HB evolution, and given that mass loss increases with stellar metallicity, we have computed HB models for a metallicity twice solar 5 . This choice allows a direct comparison with the evolutionary computations performed by Yong et al. (2000). All HB models have a $1 M_{\odot}$ RGB progenitor with an initial chemical composition of $Z=0.04$ and $Y=0.34$. The He core mass of this structure at He ignition is equal to $0.466 M_{\odot}$, while its surface He abundance in the same evolutionary phase is of the order of $Y=0.36$. The standard models, i.e. the ones computed neglecting mass loss, have been presented by Bono et al. (1997).

In the various panels of Fig. 4, a comparison between standard models and models accounting for mass loss (according to Eq. (5)) is presented. When computing the models with mass loss, we have accounted for this process along the whole evolutionary path, starting from the Zero Age Horizontal Branch (ZAHB) until an effective temperature of the order of $40000 \mathrm{~K}$ (see previous discussion). This implies that we are also using our mass loss recipe out of its validity range in luminosity.

\footnotetext{
5 All evolutionary models computed here as well as additional models can be obtained upon request.
} 


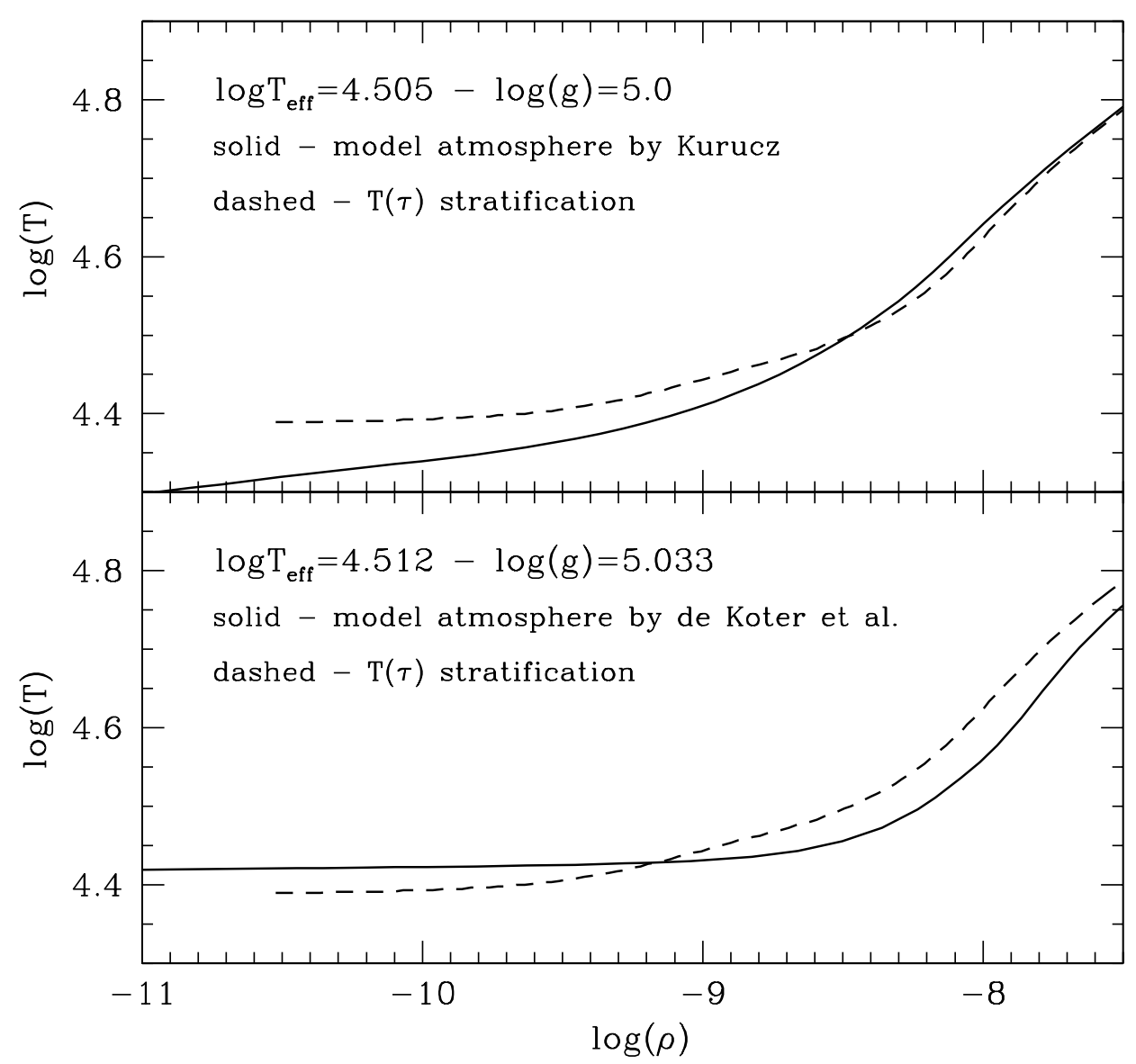

Fig. 3. Upper panel: comparison between the atmosphere thermal stratification provided by the $T(\tau)$ integration and by the model atmosphere of Kurucz (1993) for the labelled values of effective temperature and gravity. Bottom panel: as upper panel, but for the model atmospheres of de Koter et al. (1993, 1997), for the labelled values of effective temperature and gravity. In all cases, a chemical composition of $Z=0.012 ; Y=0.25$ has been adopted.
In the case of the most massive star, i.e. the coolest one, with a ZAHB location below $10000 \mathrm{~K}$, we are also slightly extrapolating Eq. (5) out of its validity range in $T_{\text {eff }}$.

We would expect that the computed mass-loss rates of the order of $10^{-12} M_{\odot} \mathrm{yr}^{-1}$ are too low to alter evolutionary tracks in a major way. Indeed, as shown in Fig. 4, the evolutionary paths of the selected models are not significantly affected by the occurrence of mass loss at the computed rates. The less massive, hottest model has lost an amount of mass of $2.2 \times 10^{-4} M_{\odot}$ at the end of the He central burning phase, while the coolest model, the one with mass equal to $0.530 M_{\odot}$, has lost $2.4 \times 10^{-4} M_{\odot}$ at the end of the same evolutionary phase. The amount of mass lost is slightly larger for the more massive model since this model is brighter, and the mass-loss rate most strongly depends on $L$. From these evolutionary computations, we arrive at the following conclusions:

- mass-loss rates due to radiation pressure are not sufficient as to significantly modify evolutionary tracks for HB stars;

- the mass-loss computations do not provide the high rates needed in the Yong et al. (2000) scenario for explaining the occurrence of EHB stars in metal-rich clusters such as NGC 6791;

- since the efficiency of radiation-driven wind decreases with stellar metallicity, the effects are likely to be even smaller for HB stars in more metal-poor clusters.

\subsection{HB Evolution with increasing mass loss due to radiative levitation}

Before we present the effects of increased mass loss due to radiative levitation on $\mathrm{HB}$ evolution, we first discuss the connection between mass loss and the changes of the surface chemical abundances due to these physical processes of radiative levitation and atomic diffusion. Our calculations show that the mass-loss efficiency increases strongly when the envelope abundances of heavy elements increase as a consequence of radiative levitation. Nevertheless, it is also well known that mass loss works as a competing process to diffusion by decreasing the efficiency of radiative levitation in producing large chemical overabundances - at least for heavy elements such as silicon. In particular, Michaud \& Charland (1986) have shown that if mass loss increases beyond $10^{-14} M_{\odot} \mathrm{yr}^{-1}$, chemical overabundances could be wiped out. In more recent times, Unglaub \& Bues (2001) have investigated the influence of diffusion and mass loss on the chemical composition of sdB stars. The main outcome of their work was that observed chemical patterns can only be explained if mass-loss rates are in the range $10^{-14}<\dot{M}\left(M_{\odot} \mathrm{yr}^{-1}\right)<10^{-12}$. Higher rates would basically prevent the effects of diffusion, whereas for lower rates helium would sink in too short time scales compared to the typical lifetime of an sdB star. Note that our mass-loss predictions fall in the middle of the range of the calculations by Unglaub \& Bues (2001) for metallicities typical of GCs with extended blue 

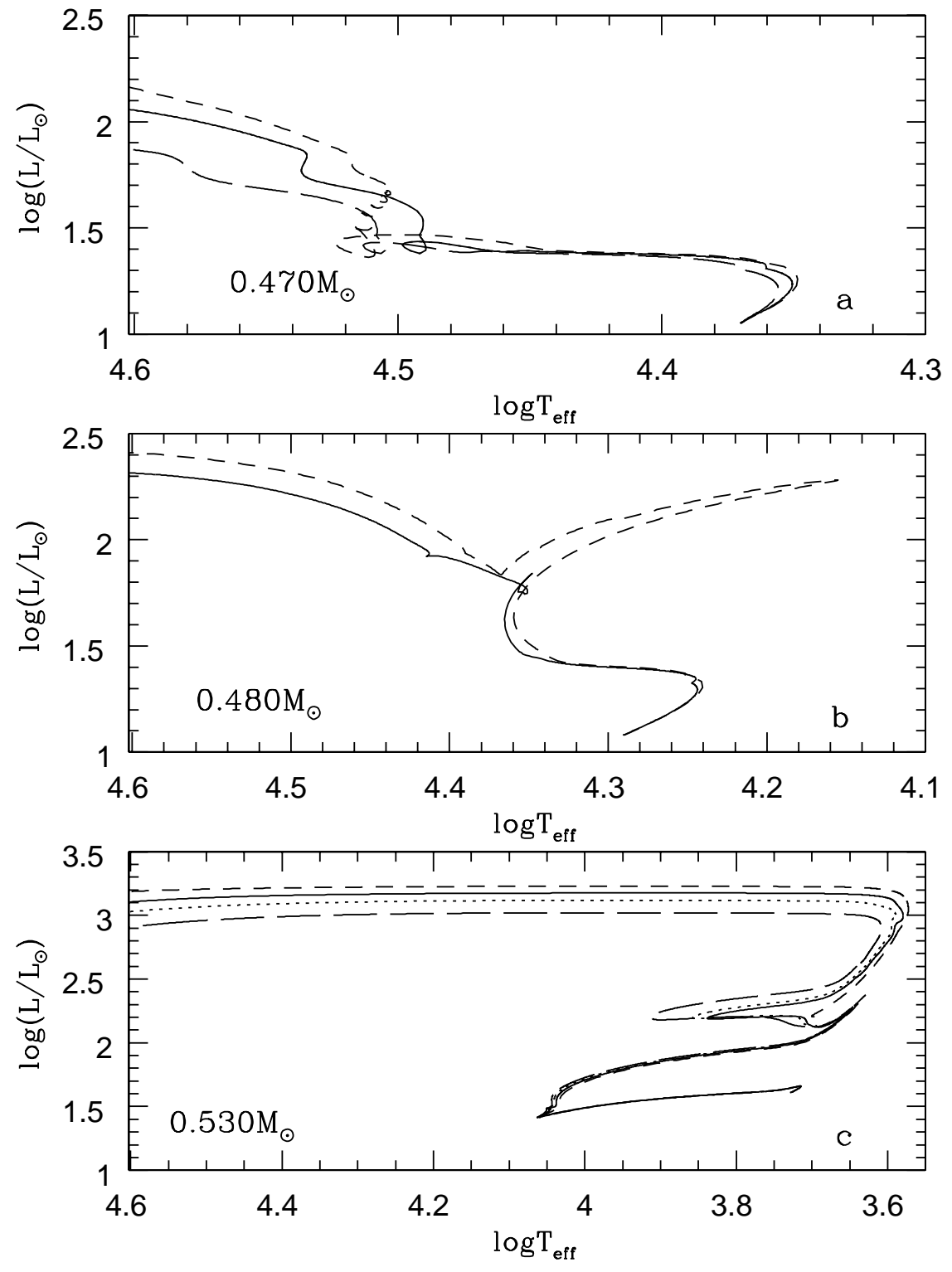

Fig. 4. The H-R diagram representing the evolution of selected HB models with various initial mass (as labelled) whose RGB progenitor mass is equal to $1 M_{\odot}$; with an initial chemical composition of $Z=0.04 ; Y=0.34$. In each panel, the short dashed line corresponds to the standard model (neglecting mass loss), while the solid line refers to models accounting for mass loss according to Eq. (5). In panels a) and c), models accounting for an enhancement of the surface metallicity (see text for details) are also plotted (dotted line $-Z=0.1$; long-dashed line $Z=0.2)$.

tails; once radiative levitation becomes effective in strongly increasing the stellar metallicity, mass loss strongly increases, and becomes of the same order of magnitude to - or even larger than - the upper limit quoted in the work of Unglaub \& Bues. If these physical processes indeed occur in real stars, they should have strong effects on the measured abundances of helium and heavy elements in hot HB stars. A detailed investigation into this topic is beyond the scope of the present work, but we wish to emphasise the further need for accurate chemical separation computations that consistently account for diffusion, radiative levitation, and reliable mass-loss predictions. This, as a function of metallicity and other stellar parameters such as mass, effective temperature and luminosity.
We now turn to the effect of increased mass loss on the evolution of HB stars. Given the importance of the metallicity on the line driving efficiency, we maximise the effects of radiative levitation on the surface abundance. This was done by simply assuming that starting from the ZAHB the surface metallicity is equal to five times or ten times the solar value. Note that we do not properly account for radiative levitation in the evolutionary code, but assume that the diffusion process is a fast and efficient mechanism to increase the surface metallicity (Michaud et al. 1985). Although this is a rather crude approach, we are only interested in checking the maximum effect of radiative levitation on the mass-loss efficiency, and we do therefore not account for possible changes in the envelope opacity properties due to 
this metallicity increase. In case we would have accounted for these effects, the models would have been somewhat fainter and cooler, which would have had the effect of slightly decreasing the mass-loss efficiency. In other words, our more crude approach has the effect of maximising the efficiency of mass loss.

The additional experiments (also shown in Fig. 4), clearly show that even with the assumed increase of the surface stellar metallicity, the mass-loss efficiency is still too low to affect the HB evolution. Therefore, it is worth noting that:

- it is almost impossible that an in situ mass loss process due to radiation pressure alone can affect the HB mass distribution in any major way;

- a relevant parameter in population synthesis studies is the maximum mass of $\mathrm{HB}$ structures that at the end of the central He-burning phase behave as AGB-manqué stars (Greggio \& Renzini 1990). Due to the low efficiency of mass loss during the HB phase, this critical mass is not affected by the inclusion of our mass loss recipe in stellar model computations.

\section{Mass loss, HB rotation rates, \& the $\log g$ jump}

In this section we discuss the implication of mass loss on HB rotational velocities and the "low gravity" problem for hot $\mathrm{HB}$ stars, which is represented by a jump in $\log g$ at $T_{\text {eff }} \simeq 11000 \mathrm{~K}$.

Observational analyses of rotational velocities of HB stars show that among the cool group $\left(T_{\text {eff }} \leq 11000 \mathrm{~K}\right)$ both fast and slow rotators are present (Peterson et al. 1995), but that for the hot group $\left(T_{\text {eff }}>11000 \mathrm{~K}\right)$ all stars rotate slowly (Behr et al. 2000a, 2000b; Recio-Blanco et al. 2002). We argue that the absence of fast rotators in HB stars hotter than $11000 \mathrm{~K}$ can be explained by a stellar wind set up by radiative levitation of heavy elements (Sweigart 2000), which could contribute significantly to the removal of angular momentum. Recio-Blanco et al. (2002) argue that the mass-loss rate may increase by a large factor between 10000 and $20000 \mathrm{~K}$ due to a change in the ionisation state of hot star winds (referring to the work by Vink et al. 2000 on massive stars). However, as we have shown in Fig. 1, dramatic changes in the mass-loss rate over $T_{\text {eff }}$ are absent for HB stars. This notwithstanding, as can be noted from Eq. (5), mass loss increases by about 2 dex when the photospheric metal abundance $(Z)$ increases by 2 dex. We therefore argue that the striking photospheric abundances in hot HB stars, which are most likely due to the onset of radiative levitation, may spin down the surface velocities of HB stars hotter than $11000 \mathrm{~K}$, explaining the absence of fast rotation at these temperatures. Note that firmer and more quantitative conclusions can only be achieved by understanding the coupling between mass loss and stellar rotation (see Soker \& Harpaz 2000).

In addition, we question whether the increase in the massloss rate around $11000 \mathrm{~K}$ invalidates the use of hydrostatic model atmospheres (such as those by Kurucz) for hot HB stars. For massive $\mathrm{O}$ stars it is a well-known fact that neglecting winds in model atmosphere calculations causes systematic errors in the derived atmospheric parameters, notably $\log g$. This has even led to a systematic discrepancy between masses derived from stellar spectra vs. those from evolutionary models, the "mass-discrepancy" (Groenewegen \& Lamers 1989; Herrero et al. 1992). Indeed, the luminosities for HB stars are much lower (having the effect of lowering the mass-loss rates), but the stars are also less massive (increasing $\dot{M}$ ), and have much smaller radii, which substantially increases the more relevant parameter, the mass flux. It is therefore not at all obvious that hydrostatic model atmospheres are applicable to these types of objects. Even more so, atmospheric analyses for hot HB stars have shown that there is also a "mass discrepancy" for these objects (Moehler et al. 1995), whereas the atmospheric determinations and canonical evolutionary models do agree for the cooler HB stars. Although the "low gravity" problem for hot HB stars may partially be explained by radiative levitation of metals (Grundahl et al. 1999; Moehler et al. 2000), it has not been solved completely (Moehler 2001), the residual discrepancy that is still left between $15000<T_{\text {eff }}<20000 \mathrm{~K}$ is about 0.1 dex in $\log g$.

To test whether stellar winds have a noticeable effect on stellar spectra of hot $\mathrm{HB}$ stars, we compute $\mathrm{H} \gamma$ line profiles for these objects using the model atmosphere code ISA-WIND and the synthetic spectrum code WYNSPEC (de Koter et al. 1997). We compare $\mathrm{H} \gamma$ line profiles for a solar metallicity star with the following stellar parameters: $T_{\text {eff }}=17500 \mathrm{~K}, M=0.56 M_{\odot}$, and $\log \left(L / L_{\odot}\right)=1.37$, corresponding to $\log g=4.73$. Using Eq. (5), we expect such a star to have a mass-loss rate $\log \dot{M}$ $\left(M_{\odot} \mathrm{yr}^{-1}\right)=-11.85$, and we therefore use this value in the computation of an $\mathrm{H} \gamma$ line profile, and compare this line profile to a model with negligible mass loss (we choose a rate typical for the Sun: $\left.\log \dot{M}\left(M_{\odot} \mathrm{yr}^{-1}\right)=-14\right)$.

As can be seen in the upper panel of Fig. 5 wind emission in the model with significant mass loss (dashed line) has a noticeable effect on the line wings, which may mimic a lower $\log g$ from Balmer $\mathrm{H} \gamma$ line profiles. To check whether the $\log g$ jump could indeed be an artifact of the use of hydrostatic model atmospheres, we compare $\mathrm{H} \gamma$ line profiles for models with different $\log g$ values: $\log g=4.73$, vs. a model with $\log g=4.63$ (or equivalently a mass of $M=0.44 M_{\odot}$ ), and keep all other parameters fixed. This comparison is also shown in Fig. 5. The bottom panel shows $\mathrm{H} \gamma$ for the high gravity (solid line) and the low gravity (dashed line) model. As expected, Stark effects broaden the line wings for the higher gravity model.

Although the effects in the two panels of Fig. 5 are small, they yield the same systematic trend. They are so similar that it suggests that the neglect of winds in atmospheric analyses of hot HB stars can mimic too low surface gravities. An increase of mass loss due to the increased surface abundances of hot HB stars may therefore invalidate the use of hydrostatic atmospheres for these stars. Note that the use of such models is considered robust for the cooler HB models, where indeed no discrepancy between evolutionary and spectroscopic masses has been reported. We argue that the "wind emission" effect - together with the metal enhancement due to radiative levitation - is the most likely explanation for the observed jump in $\log g$.

We then arrive at the following scenario for the "zoo" of problems in $\mathrm{HB}$ morphology around $11000 \mathrm{~K}$ : due to the more stable atmospheres of the hotter HB stars radiative 

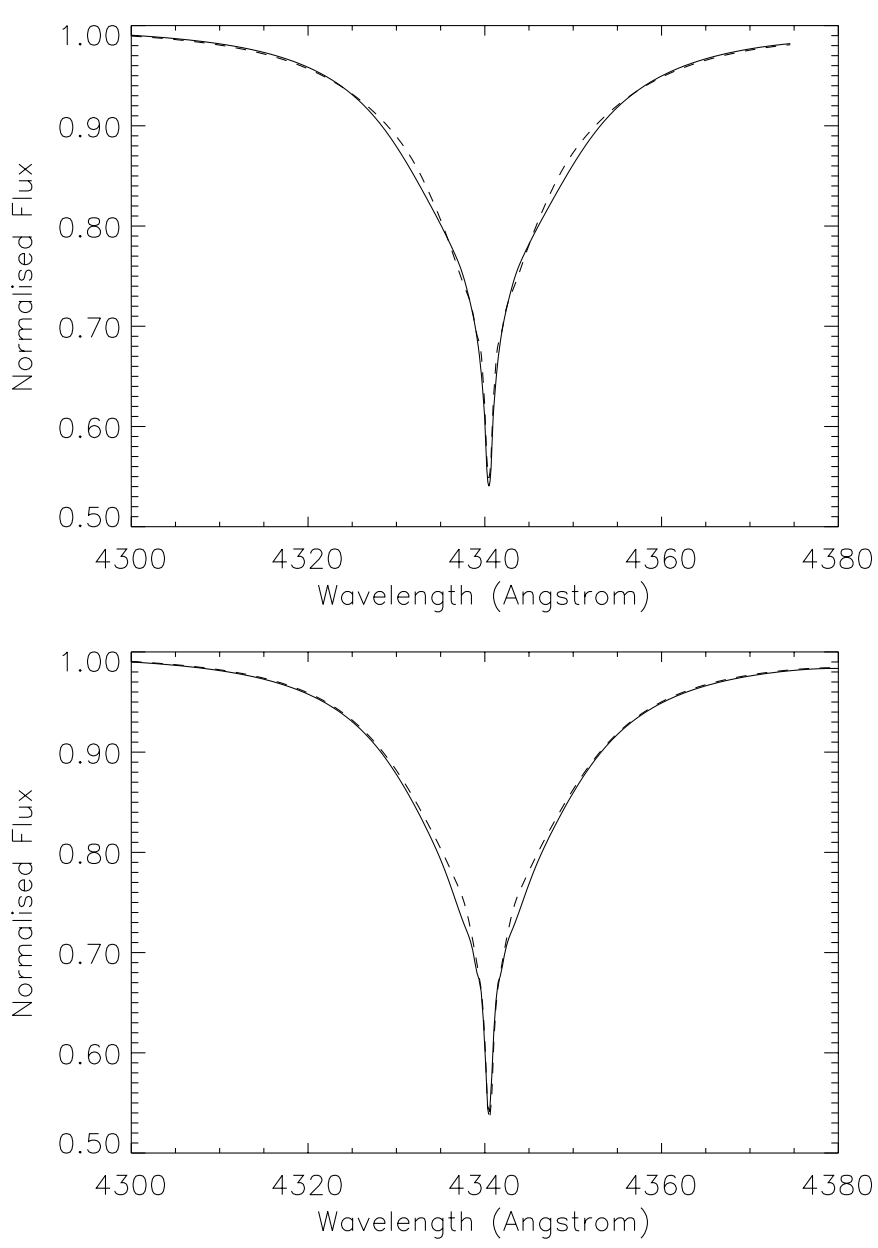

Fig. 5. Upper panel: the normalised flux at $\mathrm{H} \gamma$ for a model with an expected mass-loss rate of $\log \dot{M}\left(M_{\odot} \mathrm{yr}^{-1}\right)=-11.85$ (dashed line), and a model for negligible mass loss, i.e. $\log \dot{M}\left(M_{\odot} \mathrm{yr}^{-1}\right) \simeq-14$ (solid line). Bottom panel: a comparison for the high gravity $(\log g=4.73$; solid line) and the low gravity $(\log g=4.63$; dashed line) model. The other stellar parameters in both panels are: $T_{\text {eff }}=17500 \mathrm{~K}, \log \left(L / L_{\odot}\right)=$ 1.37 , and solar metallicities.

levitation increases the metal abundances for these objects. This subsequently explains: (i) the striking surface abundances in hot HB stars; (ii) the existence of the Strömgren $u$-jump. It also reduces the $\log g$ discrepancy. As the abundance spectroscopic determinations indicate that only the hotter HB models are affected by radiative levitation, a stellar wind is expected to be set up with an increased $\dot{M}$ by two orders of magnitude. This onset of (significant) mass loss can then also help in explaining (iii) the absence of fast rotators for HB stars, and (iv) the jump in $\log g$. In passing, we finally note that (v) the gap observed along the $\mathrm{HB}$ of many $\mathrm{GCs}$ at $(B-V) \simeq 0\left(T_{\mathrm{eff}} \simeq 9000 \mathrm{~K}\right)$ could also be due to an atmospheric phenomenon related to the chemical peculiarities induced by radiative levitation as suggested by Caloi (1999).

\section{Conclusions and outlook}

In this paper we have, for the first time, computed mass-loss rates for HB stars. We have shown that the computed rates, as predicted by the most plausible mechanism of radiation pressure on spectral lines, are too low to produce $\mathrm{EHB} / \mathrm{sdB}$ stars. This invalidates the scenario outlined by Yong et al. (2000) to create these objects by excessive mass loss on the HB. We argue, however, that mass loss plays a role in the distribution of rotational velocities of hot HB stars, and for the so-called $\log g$ jump. The mass loss recipe derived in this paper is, strictly speaking, only valid for HB stars, but as there are hardly any mass-loss predictions available for low-mass blue stars, the recipe may also be applied to: post-HB, AGBmanqué, UV-bright stars, extreme helium stars, as long as the desired accuracy is within a factor of two, and as long as the effective temperatures are not higher than $40000 \mathrm{~K}$.

Although we have proposed a scenario where winds are ubiquitous for hot HB stars, and subsequently affect the rotational velocities, as well as the atmospheric parameters $(\log g)$, there is still a lot of work to be done.

First and foremost, spectral evidence for mass loss in $\mathrm{HB}$ and sdB stars ought to be sought to check whether the massloss rates, as derived in this paper, indeed occur. Diffusion calculations including mass loss for sdB stars (Unglaub \& Bues 2001) suggest that our derived mass-loss rates are reasonable, but this is certainly not a model-independent check.

Second, evolutionary models including rotation (first steps have been undertaken by Sills \& Pinsonneault 2000) and mass loss should be computed to see whether the absence of fast rotators for stars hotter than $11000 \mathrm{~K}$ can indeed be due to the removal of angular momentum due to stellar winds.

Last but not least, systematic atmospheric analyses of hot HB stars accounting for the actual surface heavy elements distribution and including mass loss should be performed to see whether the $\log g$ jump is indeed an artifact of the adopted hydrostatic model atmospheres. The current situation, where evolutionary models are not in agreement with the spectral analyses is highly undesirable, as this suggests that current stellar evolution theory is not only incapable of producing extreme HB stars, but that even "normal" blue HB stars pose a serious problem. In other words, a solution to the "low gravity" problem for hot HB stars could significantly enhance our current understanding of the later phases of stellar evolution.

Acknowledgements. We would like to thank Allen Sweigert and Marcio Catelan for constructive comments that helped improve the paper. We would additionally like to thank Giuseppe Bono and Rubina Kotak for fruitful discussions, Noam Soker for a swiftly produced referee report, and Alex de Koter and Robert Kurucz for the use of their model atmospheres. SC acknowledges financial support by MURST-Cofin2002.

\section{References}

Abbott, D. C., \& Lucy, L. B. 1985, ApJ, 288, 679

Anders, E., \& Grevesse, N. 1989, Geochim. Cosmochim. Acta, 53, 197

Audouze, J. 1987, Observational Cosmology, ed. A. Hewitt et al. (Reidel Publ.), IAU Symp., 124, 89

Babel, J. 1996, A\&A, 309, 867

Behr, B. B., Cohen, J. G., McCarthy, J. K., \& Djorgovski, S. G. 1999, ApJ, 517, L135

Behr, B. B., Djorgovski, S. G., Cohen, J. G., et al. 2000a, ApJ, 528, 849 
Behr, B. B., Cohen, J. G., \& McCarthy, J. K. 2000b, ApJ, 531, L37

Bergeron, P., Wesemael, F., Michaud, G., \& Fontaine, G. 1988, ApJ, 332, 964

Bono, G., Caputo, F., Cassisi, S., Castellani, V., \& Marconi, M. 1997, ApJ, 489, 822

Brown, T. M., Sweigart, A. V., Lanz, T., Landsman, W. B., \& Hubeny, I. 2001, ApJ, 562, 368

Caloi, V. 1999, A\&A, 343, 904

Cassisi, S., \& Salaris, M. 1997, MNRAS, 285, 593

Castellani, V., Ciacio, F., Degl'Innocenti, S., \& Fiorentini, S. 1997, AAP, 322, 801

Castor, J. I., Abbott, D. C., \& Klein, R. I. 1975, ApJ, 195, 157

Catelan, M., Borissova, J., Sweigart, A. V., \& Spassova, N. 1998, ApJ, 494, 265

Catelan, M., Bellazzini, M., Landsman, W. B., et al. 2001, AJ, 122, 317

de Koter, A., Schmutz, W., \& Lamers, H. J. G. L. M. 1993, A\&A, 277, 561

de Koter, A., Heap, S. R., \& Hubeny, I. 1997, ApJ, 477, 792

Dorman, B., O'Connell, R. W., \& Rood, R. T. 1995, ApJ, 442, 105

Ferraro, F. R., Paltrinieri, B., Fusi Pecci, F., Rood, R. T., \& Dorman, B. 1998, ApJ, 500, 311

Fusi Pecci, F., Ferraro, F. R., Bellazzini, et al. 1993, AJ, 105, 1145

Glaspey, J. W., Michaud, G., Moffat, A. F. J., \& Demers, S. 1989, ApJ, 339,926

Green, R. F., Schmidt, M., \& Liebert, J. 1986, ApJS, 61, 305

Greenstein, J. L. 1971, Proc. IAU Symp. 42., ed. W. J. Luyten (Springer-Verlag, Dordrecht), 46

Greggio, L., \& Renzini, A. 1990, ApJ, 364, 35

Groenewegen, M. A. T., \& Lamers, H. J. G. L. M. 1989, A\&AS, 79, 359

Grundahl, F., Catelan, M., Landsman, W. B., Stetson, P. B., \& Andersen, M. I. 1999, ApJ, 524, 242

Hamann, W.-R., Gruschinske, J., Kudritzki, R. P., \& Simon, K. P. 1981, A\&A, 104, 249

Heber, U., Moehler, S., Napiwotzki, R., Thejll, P., \& Green, E. M. 2002, A\&A, 383, 938

Herrero, A., Kudritzki, R. P., Vilchez, J. M., et al. 1992, A\&A, 261, 209

Howarth, I. D. 1987, MNRAS, 225, 33

Kilkenny, D., Koen, C., O’Donoghue, D., \& Stobie, R. S. 1997, MNRAS, 285, 640

Koopmann, R. A., Lee, Y.-W., Demarque, P., \& Howard, J. M. 1994 , ApJ, 423, 380

Krishna-Swamy, K. S. 1966, ApJ, 145, 174

Krticka, J., \& Kubát, J. 2000, A\&A, 359, 983

Kurucz, R. L. 1993, SAO CD-ROM

Lamers, H. J. G. L. M., \& Cassinelli, J. P. 1999, Introduction to stellar winds (Cambridge Univ. Press)

Lee, Y-.W., Demarque, P., \& Zinn, R. 1994, ApJ, 423, L248

Lucy, L. B. 1987, in ESO Workshop on the SN 1987A, Proceedings (A88-35301 14-90), 417

Lucy, L. B. 1999, A\&A, 345, 211

Lucy, L. B., \& Abbott, D. C. 1993, ApJ, 405, 738
Lucy, L. B., \& Solomon, P. 1970, ApJ, 159, 879

Mengel, J. G., Norris, J., \& Gross, P. G. 1976, ApJ, 204, 488

Michaud, G., \& Charland, Y. 1986, ApJ, 311, 326

Michaud, G., Bergeron, P., Wesemael, F., \& Fontaine, G. 1985, ApJ, 299, 741

Moehler, S. 2001, PASP, 113, 1162

Moehler, S., Heber, U., \& de Boer, K. S. 1995, A\&A, 294, 65

Moehler, S., Sweigart, A. V., Landsman, W. B., \& Heber, U. 2000, A\&A, 360, 120

Newell, E. B. 1973, ApJS, 26, 37

Owocki, S. P., \& Puls, J. 1999, ApJ, 510, 355

Pagel, B. E. J., Simonson, E. A., Terlevich, R. J., \& Edmunds, M. G. 1992, MNRAS, 255, 325

Pauldrach, A. W. A., Puls, J., Kudritzki, R. P., Méndez, R. H., \& Heap, S. R. 1988, A\&A, 207, 123

Peterson, R. C., Rood, R. T., \& Crocker, D. A. 1995, ApJ, 452, 214

Piotto, G., Zoccali, M., King, I. R., et al. 1999, AJ, 118, 1727

Porter, J. M., \& Drew, J. E. 1995, A\&A, 296, 761

Puls, J., Kudritzki, R.-P., \& Herrero, A. 1996, A\&A, 305, 171

Raimondo, G., Castellani, V., Cassisi, S., Brocato, E., \& Piotto, G. 2002, ApJ, 569, 975

Recio-Blanco, A., Piotto, G., Aparicio, A., \& Renzini, A. 2002, ApJL, in press [astro-ph/0204403]

Rich, R. M., Sosin, C., Djorgovski, S., et al. 1997, ApJ, 484, L25

Sandage, A., \& Wallerstein, G. 1960, ApJ, 131, 598

Sandage, A., \& Wildey, R. 1967, ApJ, 150, 469

Sills, A., \& Pinsonneault, M. H. 2000, ApJ, 540, 489

Soker, N. 1998, AJ, 116, 1308

Soker, N., \& Harpaz, A. 2000, MNRAS, 317, 861

Soker, N., Catelan, M., Rood, R. T., \& Harpaz, A. 2001, ApJ, 563, L69

Springmann, U. W. E., \& Pauldrach, A. W. A. 1992, A\&A, 262, 515

Sweigart, A. V. 1997, ApJ, 474, L23

Sweigart, A. V. 2000, in Mixing and Diffusion in Stars: Theoretical Predictions and Observational Constraints, 24th meeting of the IAU, Joint Discussion [astro-ph/0103133], in press

Sweigart, A. V., Brown, T. M., Lanz, T., Landsman, W. B., \& Hubeny, I. 2002, Proceeding of the Workshop, Omega Centauri: a Unique Window into Astrophysics (Cambridge, August, 2001), ASP Conf. Ser., ed. F. van Leeuwen, G. Piotto, \& J. Hughes [astro-ph/0203063], in press

Unglaub, K., \& Bues, I. 2001, A\&A, 374, 570

van den Bergh, S. 1967, AJ, 72, 70

Vink, J. S., \& de Koter, A. 2002, A\&A [astro-ph/0207170], in press

Vink, J. S., de Koter, A., \& Lamers, H. J. G. L. M. 1999, A\&A, 350, 181

Vink, J. S., de Koter, A., \& Lamers, H. J. G. L. M. 2000, A\&A, 362, 295

Vink, J. S., de Koter, A., \& Lamers, H. J. G. L. M. 2001, A\&A, 369, 574

Willson, L. A., \& Bowen, G. H. 1984, Nature, 312, 429

Yong, H., Demarque, P., \& Yi, S. 2000, ApJ, 539, 928

Zoccali, M., Cassisi, S., Bono G., et al. 2000, ApJ, 538, 289 